\title{
8. Evidence from Spectra of Bright Fireballs
}

\author{
ZDeñ̌k Ceplecha \\ Astronomical Institute of the Czechoslovak Academy of Sciences \\ Ondřejov, Czechoslovakia
}

\begin{abstract}
Spectral data with dispersions from 11 to $94 \AA / \mathrm{mm}$ on 4 fireballs of actual brightness of -4 to -12 magnitude and with velocities of about $30 \mathrm{~km} / \mathrm{s}$ at 70 to $80 \mathrm{~km}$ heights are used for studies of meteor radiation problems. Previously published analyses need revision for two main reasons: (a) the absolute values of oscillator strengths of Fe I lines from laboratory data were recently recognized to be 1 order of magnitude lower, $(b)$ the luminous efficiency factor $\tau$ of $F e I$ is now much better known from several different experiments. The radiation of fireballs is found to be strongly affected by self-absorption. But if the emission curve of growth is used for correction of the self-absorption of Fe I lines, a great discrepancy between spectral data and efficiency data for total Fe I light is found. If one assumes that the self-absorption is superposed on another effect, a decrease of the dimensions of the radiating volume with increasing lower potential $\mathrm{E}_{1}$, the spectral data on Fe I lines will be in agreement with the luminous efficiency of total Fe I meteor radiation. Formulas for emission curve of growth and Boltzmann distribution including this effect are derived. This effect is important for fireballs brighter than about -1 or -2 magnitude, while self-absorption seems to be important even for fainter meteors. The optically thin radiation of all $F e I$ lines might be expected for meteors fainter than +5 magnitude. Excitation temperature of $5500^{\circ} \mathrm{K}$ and relaxation time of $0.02 \mathrm{~s}$ were found as typical values for the Fe I radiation of fireballs studied. The light of fireballs is emitted during a relatively long relaxation time, which is many orders of magnitude longer than the time necessary for spontaneous radiation of excited $F e I$ atoms. The dimensions of the radiating volume of $F e I$ gas for lines with $\mathrm{E}_{1}=0$ were found to be $0.3 \times 9 \mathrm{~m}$ at 0 absolute magnitude and $2 \times 60 \mathrm{~m}$ at -10 absolute magnitude. It was not possible to determine any realistic abundances of other elements due to small numbers of lines for an analysis independent of Fe I, while the Fe I curve of growth cannot be used for other elements, because the radiation originates mainly from the effective surface of the radiating volume. A general formula for meteor radiation is also derived and compared with the conventional luminosity equation.
\end{abstract}

$\mathrm{T}$ HERE IS ENOUGH LIGHT available to obtain spectra of bright fireballs with dispersions well below $100 \AA / \mathrm{mm}$. On the other hand, there are not so many bright fireballs which is, in part, the reason that published spectral data on line intensities are rather scarce. Preparing this paper
I used, almost exclusively, my own observational material resulting from ten years of systematic operation of three grating spectrographs employed in the registration of meteors at the Ondrejov Observatory in Czechoslovakia. On an average, about one year was necessary to get a good spec- 
trum of a fireball (over 100 spectral lines in the visible region) using these cameras. The direct double-station cameras (30 in number) provided us with all the necessary geometrical and velocity data on these fireballs. The reduction of four spectra is now completed; three of them have been published already (Ceplecha and Rajchl, 1963; Ceplecha, 1964, 1965, 1966) and one spectrum, S 1132, containing 990 spectral lines, is in print (Ceplecha, 1971).

The summary of the spectra I am using in this study is in table 1. All the objects were of approximately the same velocity, about $30 \mathrm{~km} / \mathrm{s}$. Thus the observational data presented here are to be referred to this particular velocity, to heights of 70 to $80 \mathrm{~km}$ and to an actual brightness interval of -4 to -12 absolute magnitude (the maximum absolute magnitudes are in the interval from -9 to $-12 \mathrm{mag}$ ). I also used qualitative data on one more spectrum, belonging to a fireball of approximately -18 magnitude. The reduction of this spectrum is not yet finished and only preliminary data are available. The spectrum belongs to a body with a significant terminal mass of about $600 \mathrm{~g}$, but no meteorite was found.

\section{NEED FOR REVISION OF PREVIOUS STUDIES}

Some studies of spectra in table 1 , which I published previously, need a revision from two main aspects:

(1) The absolute values of the oscillator strengths of $\mathrm{Fe} I$ lines from laboratory data were recently recognized to be one order of magnitude

TABLE 1.-List of Meteor Spectra Used for Analysis in This Paper

\begin{tabular}{|c|c|c|c|c|c|}
\hline Spectrum No. & S 6 & S 526 & S 912 & S 1132 & (S 1135) \\
\hline $\begin{array}{l}\text { Meteor No. } \\
\text { Date } \\
\text { Time (GMT) } \\
{ }^{*} M_{\text {pan }}\end{array}$ & $\begin{array}{c}130960 \\
\text { Sept. } 13,1960 \\
18^{\mathrm{h}} 59^{\mathrm{m}} \\
-10.5\end{array}$ & $\begin{array}{c}32281 \\
\text { Nov. } 13,1961 \\
21^{\mathrm{h}} 49^{\mathrm{m}} 46^{\mathrm{s}} \\
-9.6\end{array}$ & $\begin{array}{c}36221 \\
\text { Sept. } 26,1962 \\
23^{\mathrm{h}} 18^{\mathrm{m}} 16^{\mathrm{s}} \\
-10.3\end{array}$ & \multicolumn{2}{|c|}{$\begin{array}{c}38421 \\
\text { Apr. } 21,1963 \\
1^{\mathrm{b}} 13^{\mathrm{m}} 48^{\mathrm{s}} \\
-12.4\end{array}$} \\
\hline $\begin{array}{l}\text { Appearance on } \\
\text { spectral plate }\end{array}$ & flare of $0.025 \mathrm{~s}$ & $\begin{array}{l}\text { trail of } 0.6 \mathrm{~s} \text { with } \\
\text { many flares }\end{array}$ & $\begin{array}{l}\text { trail of } 0.4 \mathrm{~s} \text { with } \\
\text { steep but smooth } \\
\text { light curve }\end{array}$ & $\begin{array}{l}\text { flare of } \\
0.022 \mathrm{~s}\end{array}$ & $\begin{array}{l}\text { trail of } 0.4 \mathrm{~s} \\
\quad \text { with } \\
\text { terminal } \\
\text { flare }\end{array}$ \\
\hline $\begin{array}{l}\text { Spectral order } \\
\text { Dispersion }(\AA / \mathrm{mm})\end{array}$ & $\begin{array}{c}2 \text { to } 7 \\
38 \text { to } 11\end{array}$ & $\begin{array}{c}1+2 \\
94+47\end{array}$ & $\begin{array}{r}1 \\
58\end{array}$ & $\begin{array}{r}1 \\
57\end{array}$ & prism \\
\hline 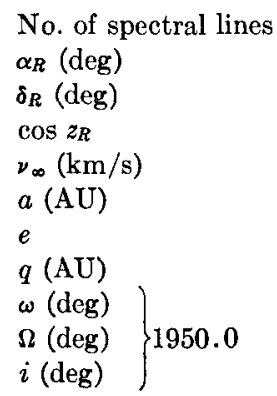 & $\begin{array}{l}166 \\
\approx 5 \\
\approx 0 \\
\approx 0.2 \\
\approx 30\end{array}$ & $\begin{array}{c}161 \\
58.2 \\
16.5 \\
0.785 \\
28.86 \\
2.30 \\
0.814 \\
0.427 \\
105.5 \\
51.1 \\
5.0\end{array}$ & $\begin{array}{l}189 \\
22.2 \\
7.1 \\
0.718 \\
31.92 \\
1.63 \\
0.849 \\
0.247 \\
129.9 \\
3.2 \\
4.9\end{array}$ & \multicolumn{2}{|c|}{$\begin{array}{c}990 \\
264.6 \\
56.8 \\
0.965 \\
32.57 \\
5.51 \\
0.819 \\
0.997 \\
190.8 \\
30.0 \\
49.0\end{array}$} \\
\hline
\end{tabular}

- $M_{\text {pan }}$ is the total absolute maximum magnitude from panchromatic plates, while magnitudes in table 2 are the actual absolute magnitudes at each point of Fe I radiation only. 
lower than previously accepted (Grasdalen et al., 1969; Garz and Kock, 1969).

(2) The luminous efficiency factor $\tau$ of $\mathrm{Fe} \mathrm{I}$ is now much better known from several different experiments (Ayers et al. 1970, Friichtenicht et al. 1968).

The absolute abundances of Fe I derived from spectral data on fireballs were previously recognized by me to be about 1 or 2 orders of magnitude greater than that derived from total Fe I light, using a luminous efficiency factor (Ceplecha, 1965, 1968). The "spectroscopic" mass of meteors studied is increased by an additional order of magnitude over the "photometric" mass, if we use the new Fe I oscillator-strength data. The main purpose of this paper is a comparison of the spectral data on $\mathrm{Fe} I$ absolute abundances with corresponding data derived from the total Fe I light curve calibrated by the luminous efficiency factor.

\section{THE LUMINOSITY EQUATION}

Before dealing with the main problem of this paper, I will derive a new general form of luminosity equation, which will help to relate the conventional luminosity equation to the gas mass in the radiating volume.

Let us denote by $m_{A}$ the total gas mass that has entered the radiating volume since the meteor beginning. Let us denote by $m_{Z}$ the total gas mass that has left the radiating volume since the meteor beginning. Then, at each instant, the total gas mass $m_{G}$ inside the radiating volume is

$$
m_{G}=m_{A}-m_{Z}
$$

Assuming that the excitation temperature and self-absorption are roughly constant during the meteor flight, which is applicable to radiation of one element in one state, we have for the intensity $I$ of the radiation:

$$
I=k m_{G}
$$

where the proportionality factor $k$ is a luminous efficiency of the radiating gas measured in ergs $\mathrm{s}^{-1} \mathrm{~g}^{-1}$. Even if $k$ is some function of time, it changes substantially less than $m_{G}$ and the proportionality, equation (2), may be considered a good approximation to the reality. This approximation is no worse than the conventional luminosity equation (4).

Substituting equation (1) in equation (2) we have

$$
I=k\left(m_{A}-m_{Z}\right)
$$

This is a meteor luminosity equation more general than the conventional equation

$$
I=-\frac{\tau}{2} v^{2} \frac{d m}{d t}
$$

Our task now is to relate the general equation (3) to the special equation (4). The additional assumptions to be applied to equation (3) can be derived from the following considerations: We differentiate (3)

$$
d I=k\left(d m_{\mathrm{A}}-d m_{\mathrm{Z}}\right)
$$

We can assume that $d m_{A}$ and $d m_{Z}$ are the same functions of time with different phase only

$$
d m_{Z}(t)=d m_{A}\left(t-t_{R}\right)
$$

where $t_{R}$ is constant: it is the time spent by an average atom inside the volume of radiating gas, and I will refer to it as the relaxation time in this paper. Now if equation (6) is substituted in equation (5) and Taylor's series with the first derivative used, we have

$$
d I=k t_{R} d\left(d m_{A} / d t\right)
$$

If we replace $m_{A}$ by the ablated meteor mass

$$
m_{A}=m_{\infty}-m
$$

and integrate, we have

$$
I=-k t_{R}(d m / d t)+k_{R}
$$

But there is practically no light at the beginning of the ablation of the meteor and $k_{R}=0$. Thus equation (9) is equivalent to equation (4), if

$$
k t_{R}=\frac{\tau v^{2}}{2}
$$

Variation in $v$ and $\tau$ during the trajectory of one meteor is neglected. We can summarize:

(1) The conventional luminous equation (4) is only a very special case of the more general equation (3).

(2) The conventional luminous equation (4) represents an average smooth change of light. 
(3) Equation (3) can easily explain the big fluctuations of the observed light curves (see fig. 1) with $d m / d t$ being a smooth function of time. If $d m_{Z}<d m_{A}, I$ increases; if $d m_{Z}>d m_{A}, I$ decreases. Thus $d m_{A}, d m_{Z}$ may be smooth functions of time, while the light intensity is flaring. Figure 1 shows this clearly even for $t_{R}=$ const.; but without this limiting assumption, the general form of equation (3) is even more sensitive for producing big changes in light emission from small smooth changes of $d m / d t$.

\section{SELF-ABSORPTION IN SPECTRA OF FIREBALLS}

I have already published studies of three spectra of meteors in table 1 using the method of emissioncurve-of-growth, which takes into account the optical thickness of individual spectral lines (Ceplecha 1964, 1965, 1967). After publication of

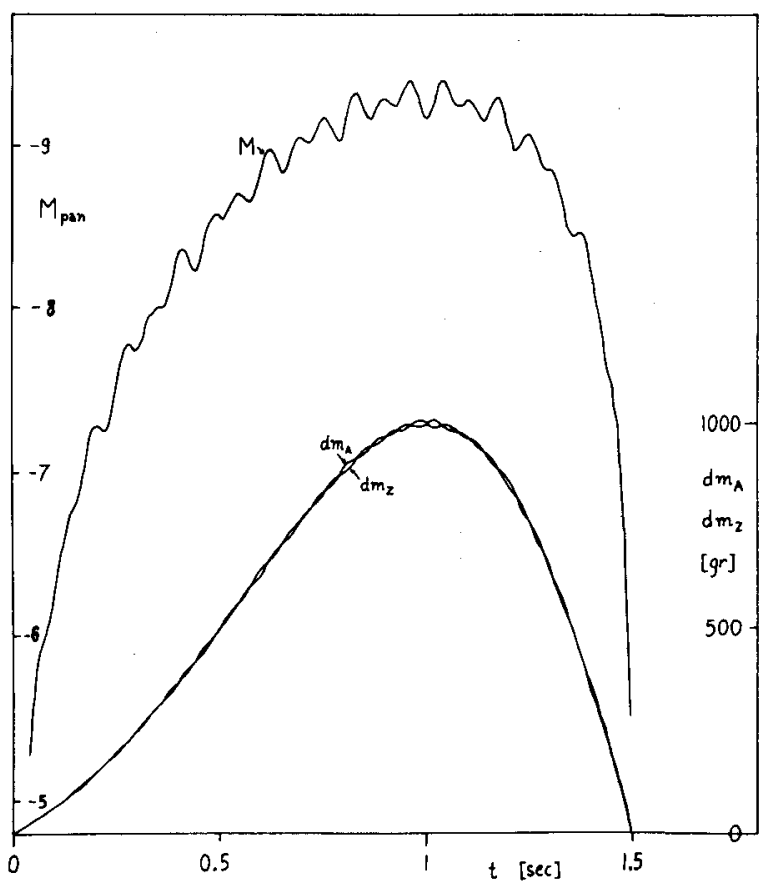

Figure 1.-Theoretical light curve computed from arbitrarily chosen but smooth values of $d m_{A}, d m_{Z}$. This shows the flaring effect. Formula (3) was used with simplifying assumption (6): $\log t_{R}=-2.7, v=30$ $\mathrm{km} / \mathrm{s}, \log k=3.42$. This is just an example representing formula (3) with limitation (6). General form of formula (3) is even more able to describe the flaring effect. this method, there were some doubts as to whether the method is applicable to fireball spectra or not. The importance of self-absorption and damping processes for fireball radiation may be well demonstrated using figure 2. Even though figure 2 is presented here only for spectrum S6, the same relative positions of individual multiplets result, using any of the other 3 spectra of table 1 at any of the measured points. The upper level potential $E_{2}$ is plotted along the x-axis and the total number of atoms of $\mathrm{Fe} I$ in all levels of the upper term, $N$ (term), is plotted along the y-axis. The observed

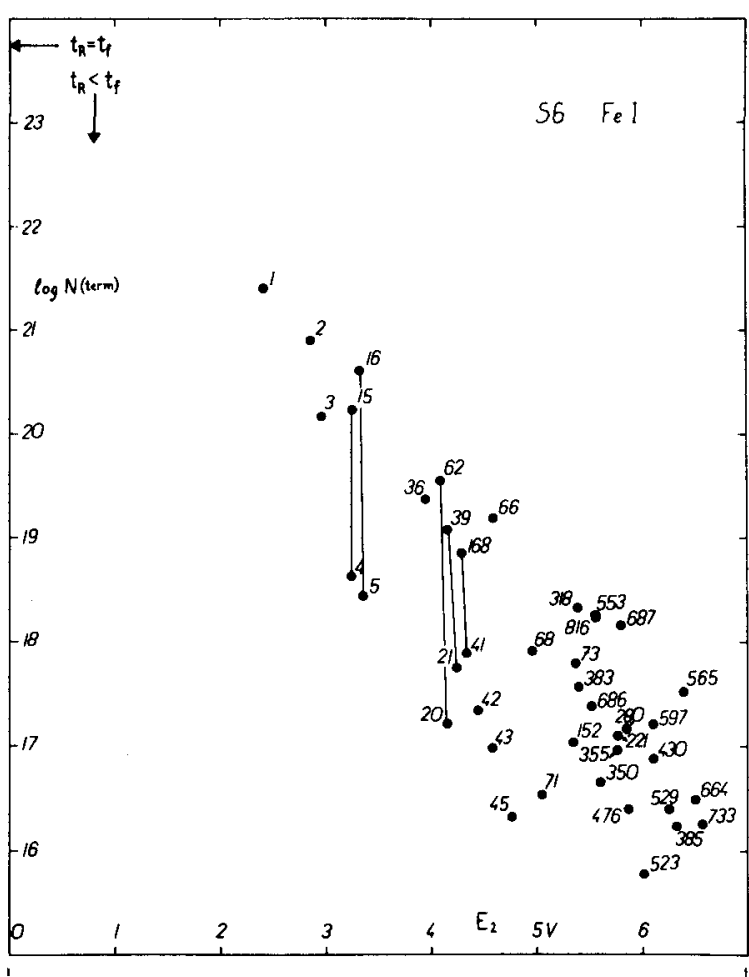

Figure 2.-Numbers of Fe I atoms, $N$ (term), occupying all levels of the upper term were computed for $S 6$ spectrum of a flare on assumption that the radiation observed in corresponding lines was optically thin $\left(I_{0}=I\right) . \log N($ term $)$ is plotted against the upper potential $E_{2}$. A straight line corresponds to Boltzmann distribution of levels. One average value for each multiplet is given. The numbering of the points corresponds to the notation of multiplets after Moore (1945). A self-absorption for multiplets with the same upper level term is evident: multiplet 4 against 15, 5 against 16,20 against 62,21 against 39,41 against 168. The parameter $t_{R}$ is the relaxation time, $t_{f}$ the duration of the flare. The extrapolation of the Boltzmann straight line to $E_{2}=0$ must be equal to or lower than the point $t_{R}=t_{f}$. 
radiation is assumed to be optically thin here, which means

$$
\log N(\text { term })=\frac{I_{0} \lambda^{3} m_{e} G_{2}}{8 \pi^{2} h e^{2} g f}
$$

$I_{0}$ is optically thin radiation: here the observed $I$ was used for figure 2 instead. $G_{2}$ is the total statistical weight of the upper term. Each point in figure 2 corresponds to an average value for the whole multiplet assuming that no self-absorption is present $\left(I_{0}=I\right)$. Some of the multiplets with more observed lines point to a dependence of $\log N$ (term) on $\log g f$ as is apparent from figure 3 . In figure 2, multiplets with the same upper level term yield greater $\log N$ (term), if their lower level is higher. Thus multiplet $4\left(E_{1}=0\right)$ shows an absorption of more than one order of magnitude relative to multiplet $15\left(E_{1}=0.9 \mathrm{~V}\right)$, multiplet 5 more absorption than multiplet 16 , multiplet 20 more than 62 , multiplet 21 more than 39 and multiplet 41 more absorption than multiplet 168 . This clearly means that we see only a part of the light produced inside the radiating gas volume and emitted in multiplets $4,5,20,21,41$. From other spectra than S6, multiplets 2, 23,
42, 43 and many others show clear evidence of self-absorption. But there is no reason for the assumption that multiplets $15,16,62,39,168$, giving the greatest population observed for the same upper level, are optically thin. If we visualize a Boltzmann distribution of levels in figure 2, we have a straight line, the slope of which corresponds to the excitation temperature $T$ :

$\log N($ term $)=-\theta E_{2}+$ const., where $\theta=\frac{5040}{T}$

The extrapolation of this straight line to $E_{2}=0$ gives the total number of atoms in the ground state, which is almost the number of all atoms of $\mathrm{Fe} I$ for all reasonable temperatures. On the other hand, spectrum $\mathbf{S} 6$ belongs to a short bright flare, the duration of which $(0.025 \mathrm{~s})$ combined with the luminous efficiency of Fe I (Ayers et al., 1970, $\left.\log \tau_{\mathrm{pan}}=-11.94\right)$ gives an upper limit for $\log N$. This is equivalent to an assumption that the duration of the flare is identical with the relaxation time $t_{R}$. If the relaxation time is less, the limit will be lower and the discrepancy higher. From figure

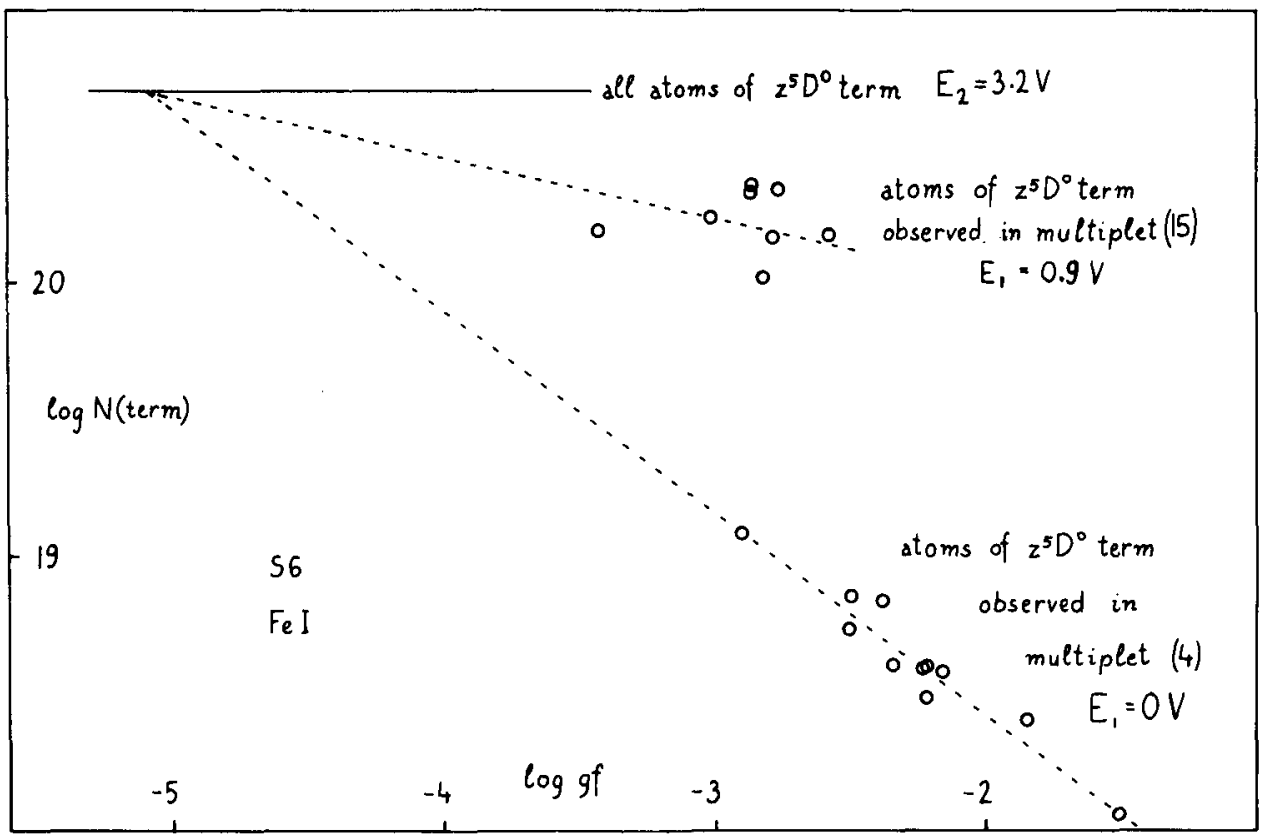

Figure 3.-Log $N$ (term), from individual lines of multiplets 4 and 15 (upper term $z^{5} D^{0}$ ), is plotted against $\log g f$ of the lines, assuming optically thin radiation as in figure 2 . Selfabsorption is evident. The dotted lines are only schematic, but indicate that lines with $\log$ $g f=-5$ and less might be expected optically thin for $z^{5} D^{0}$ of this spectrum. 
2, it is also evident that multiplets giving the greatest $\log N$ (term) must be affected by some further absorption effect. As a byproduct we can guess that the relaxation time $t_{R}$ of $\mathrm{Fe} \mathrm{I}$ lines with $E_{1}=0$ is of the order of $10^{-2}$ or $10^{-3} \mathrm{~s}$, if reasonable excitation temperatures are used. There is no reasonable way to make $t_{R}$ less than $10^{-4} \mathrm{~s}$. This time is by orders of magnitude longer than the time necessary for spontaneous radiation of the corresponding excited states of the Fe I atoms. Some quasiequilibrium conditions may be reached during this relatively long time. There is no doubt, inspecting figure 2, that self-absorption is at least partly responsible for the observed effects.

But if we use the emission curve of growth to correct for self-absorption, we get a relaxation time, $t_{R}$, still greater than the duration of the flare. I tried to use a model with exponential decrease of temperature along the radiating volume to avoid this discrepancy, but it was not much help. This suggests that another additional effect is present.

The observed radiation and deduced number of atoms are related to the whole volume of radiating gas. As we know that the wake phenomenon is strongly dependent on the excitation, we may expect the same dependence also for the "head" radiation. Then we actually do see the radiation of different spectral lines belonging to different volumes of radiating gas with different relaxation times. The low potential lines perhaps radiate from a larger volume with longer relaxation time. I will present here a simple theory of this effect and derive corresponding changes of the emission curve of growth and of the Boltzmann distribution. I will apply the theoretical results to the spectra of table 1 .

\section{EMISSION CURVE OF GROWTH WITH RESPECT TO CHANGES OF THE VOLUME OF RADIATING GAS WITH THE $E_{1}$ POTENTIAL}

I will use the same simple model of radiating gas volume that I published previously (1964), which is a cylindrical volume with great density on the surface, termed the "cylindrical shockwave" model. I assume that the length $\ell$ and radius $R$ of the cylindrical volume are proportional, $p$ being the proportionality factor. Then

$$
\ell=p R
$$

The intensity of the wake phenomenon seems to depend on the lower level $E_{1}$ of the corresponding line. Thus $\mathrm{I}$ assumed a dependence of $\ell$ and $R$ on the lower level $E_{1}$ having an exponential form

$$
\ell\left(E_{1}\right)=\ell(0) \exp \left[-2 q \chi_{1} /\left(k_{B} T\right)\right]
$$

where $\chi_{1}$ is the energy of the lower level (potential $E_{1}$ ) and $q$ is an exponential factor describing the decrease of the length of the radiating volume with increasing lower potential. Here $\ell(0)$ is the length of the radiating volume corresponding to the ground state. Following equation (18) of my previous paper (1964), we have

$$
\log N\left(E_{1}\right)=\log N(0)-5 q \Theta E_{1}
$$

for the total number of all atoms of the ground state inside a volume corresponding to the $E_{1}$ level. Here $N(0)$ is the total number of atoms of the ground state inside the radiating volume belonging to the ground state.

The Boltzmann distribution for this case is then represented by

$$
\log \frac{I_{0} \lambda^{3}}{g f}=-\Theta\left(E_{2}+5 q E_{1}\right)+\log \frac{8 \pi^{2} h e^{2}}{m_{e} U}+\log N(0)
$$

where $I_{0}$ is the optically thin intensity radiated by the assumed changing volume in all directions. The observed intensities $I$ are to be converted to $I_{0}$ by using the emission curve of growth given in our partial case by

$$
\begin{aligned}
& \log \frac{I}{\lambda B_{\lambda}}+4 q \ominus E_{1} \\
& +\log \frac{c}{8 \pi^{2} v_{m}\left[R^{2}(0)+R(0) \ell(0)\right] \cos \alpha}=\log F(X)
\end{aligned}
$$

where

$$
\begin{aligned}
& \log X=\log g \Omega-\Theta E_{1}-q \Theta E_{1} \\
& +\log \frac{3 e^{2} N(0)}{16 m_{e} c U v_{m}\left[R^{2}(0)+R(0) \ell(0)\right] \cos \alpha}
\end{aligned}
$$

and the function $F(X)$ describes the actual shape of the curve of growth. This is the same function as previously published [Ceplecha, 1964, equation (22) ]. The damping part of the emission curve of growth is also well described by 


$$
\lim _{x \rightarrow \infty} \log F(X)=1 / 2 \log X+\text { const. }
$$

in this case and generally.

The procedure used for application of the above derived formulas to the observed spectral lines is exactly the same as published previously (Ceplecha, 1964). The results of the computations are: the excitation temperature $T$, the number of all atoms of one element in one state inside a radiating volume corresponding to $E_{1}=0$, and $Y_{1 \mathrm{im}}$ proportional to the surface of this volume. A set of these values is obtained for each chosen value of $q$. If the ratio $p$ of $\ell$ to $R$ is assumed, the volume of radiating gas for $E_{1}=0$ is determined and the terminal density $n_{0}\left(\delta_{0}\right)$ at the end of the cylindrical volume as well as the average density $\bar{n}(\bar{\delta})$ may be computed. The whole procedure of numerical computations was programmed for the Minsk-22 digital computer.

We have actually added one more parameter, $q$, to be determined from observation. But this parameter may be guessed from the spectra of flares of short duration. If $d m / d t$ is determined from equation (4), and compared with the results from the emission curve of growth, we can determine the relaxation time

$$
t_{R}=\frac{m_{G}}{d m / d t} \quad m_{G}=\mu N
$$

Here $m_{G}$ is determined from the curve of growth and $d m / d t$ from the light curve. We have an additional condition for short duration flares

$$
t_{R} \leq t_{f}
$$

where $t_{f}$ is the duration of the flare.

\section{RESULTS FOR FE I}

The actual solution of the problem was possible for $\mathrm{Fe} I$ lines almost exclusively. Any other element, except for $\mathrm{Na}$ I, Ca I and $\mathrm{O}$ I in spectrum S 1132, had not a sufficient number of spectral lines for such a solution. Only the lines without any blends were used for all the computations. The $g f$-values used were from Corliss and Bozman (1962) and Corliss and Warner (1964), but corrected by -1.00 in the $\log$ to the newly established approximate absolute scale (Grasdalen et al., 1969; Garz and Kock, 1969). The excitation potentials were taken from Moore (1945).
I started with S 6 and S 1132 and found a temperature of $5500^{\circ} \mathrm{K}$ as the lowest possible to satisfy the equation (20) condition in both spectra. With this temperature, $t_{R}$ of $\mathrm{S} 6$ is equal to the duration of the flare of S 6 , and $t_{R}$ of $\mathrm{S} 1132$ is about $1 / 5$ of the duration of the flare of $S 1132$. Any temperature higher than $5500^{\circ} \mathrm{K}$ may be a solution of the problem as well, but there is another rough estimation possible using the computed density inside the radiating volume: the terminal $n_{0}$ should not exceed too much the density of the surrounding air. If $n_{0}$ of $\mathrm{Fe} I$ is not greater than 10 times the surrounding air, the temperature should not exceed some $8000^{\circ} \mathrm{K}$. If $n_{0}$ is of the order of the surrounding air, we arrive at about $5000^{\circ}$ to $6000^{\circ} \mathrm{K}$. Thus $5500^{\circ} \mathrm{K}$ was taken as a standard temperature and all the solutions for $q$ were made with this temperature. A graphical example of one solution for S 1132 is given in figures 4 and 5 . The curve of growth, figure 4, and the Boltzmann distribution, figure 5, are used simultaneously for the temperature determination. This means that the actual position of the theoretical curve of growth and the Boltzmann straight line are dependent on the positions of points in both figures 4 and 5 .

All points of the meteor spectra of table 1 were used for the solution of $q$ for Fe I lines, using the above estimated temperature. The following values were determined and are listed in table 2; number of all atoms of $\mathrm{Fe} \mathrm{I}$ inside the radiating volume; $Y_{\text {lim }}$ proportional to the surface of the radiating volume; $d m / d t$ from the light curve of total Fe I radiation $\left(\log \tau_{\mathrm{pan}}=-11.94\right.$ was used for $I$ being in 0 magnitude units combined with c.g.s.; Ayers et al., 1970); the ratio of Fe I radiation to total radiation; the relaxation time $t_{R}$; rough guesses for the ratio $p$ of $\ell$ to $R$; characteristic velocity $v_{m}$; the terminal densities $n_{0}$, $\delta_{0}$ and the average densities $n, \delta$ of $\mathrm{Fe}$ I atoms.

The most important result is that using equations (16) to (18), which describe the combination of self-absorption and changing volume of radiating gas in a simple model, we are able to put the spectral data on Fe I lines in agreement with the luminous efficiency of the total Fe I meteor radiation. If temperatures are close to $5500^{\circ} \mathrm{K}$, the values of $q$ are from 0.010 to 0.068 , depending partly on the total brightness. Extrapolating to fainter meteors, there is clear cvidence that the 


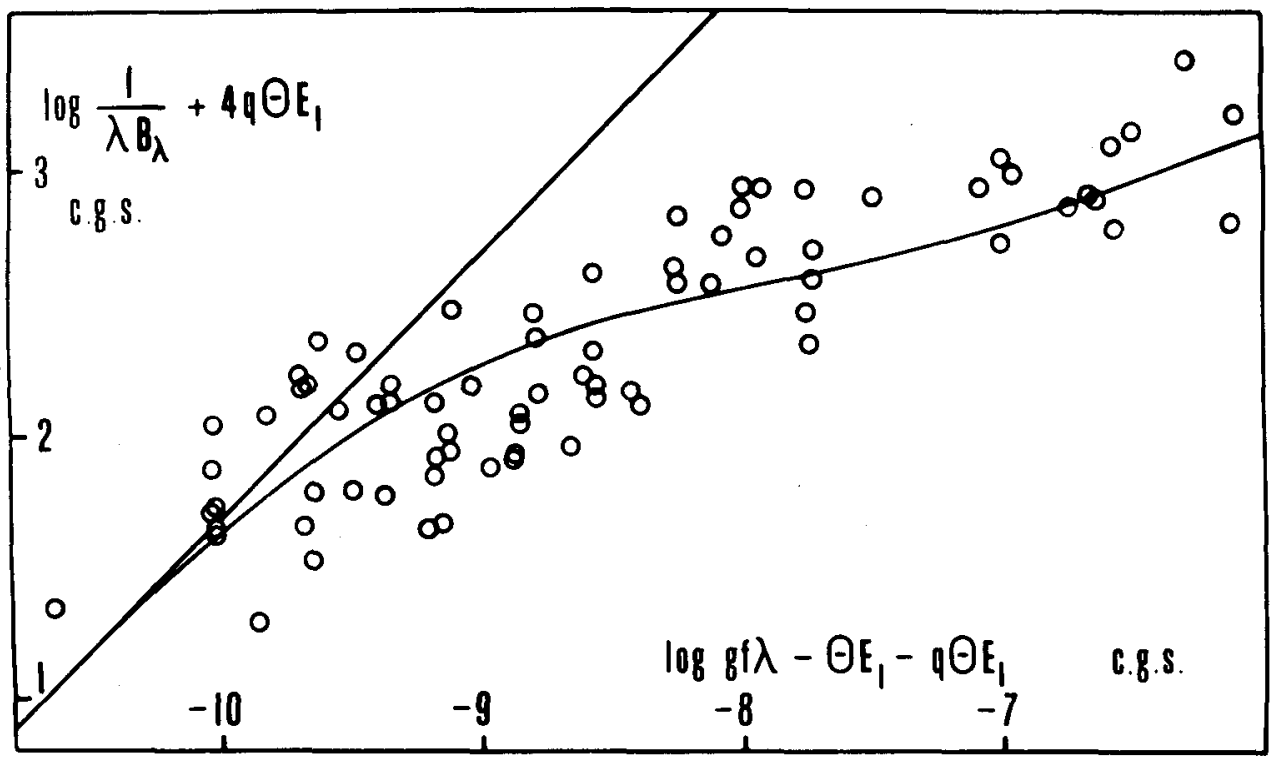

FIgure 4.-Spectrum S 1132: The emission curve of growth with respect to the decrease of volume of radiating gas with the $E_{1}$ potential. The position of the theoretical curve of growth depends on the Boltzmann distribution presented in figure 5.

effect of a change of the radiating volume with $E_{1}$ potentials may be negligible for meteors fainter than about -2 or -1 absolute magnitude. On the other hand, the self-absorption effect is important even for fainter meteors. If we compare multiplet 4 with multiplet 15 for the fireballs of table 1, and also for the relatively fainter object of -1.5 magnitude published by Harvey (1970), we see that the relative self-absorption in bright lines of multiplet 4 decreases from about $\Delta \log I=1.5$ at -10 magnitude to about $\Delta \log I=0.8$ at 0 magnitude. Extrapolating, we would expect optically thin radiation in multiplet 4 for fainter meteors than +5 magnitude, but possibly starting with even fainter objects. As multiplet 4 is one of the most affected by self-absorption, the meteor radiation in the 0 to +5 magnitude interval may be optically thin for the majority of $\mathrm{Fe} I$ lines, that is lines with $E_{\mathrm{i}} \neq 0$ and sufficiently small $g f$ values. But this very preliminary statement should be checked in each particular case in future studies.

The resulting relaxation times are in the interval $-2.58 \leq \log t_{R} \leq-1.15$, most of the values being quite close to an average value of $\log t_{R}=-1.7$. This is independent of the actual brightness or any other changing parameters. Thus the relaxation time of $0.02 \mathrm{~s}$ seems to be a typical value for $30 \mathrm{~km} / \mathrm{s}$ fireballs in the -4 to -12 absolute magnitude interval. The light of fireballs is emitted during a relatively long relaxation time of the gas. This is essentially the same result as found by Harvey (1970) for the spectrum of a -1.5 magnitude meteor.

Even if we are working with the absolute values of light intensities, it is an advantage that the computed values of the relaxation time are not dependent on absolute photometry [see equation (19) ]. They reflect the ratio of the luminous efficiency factor to absolute $g f$-values, using relative photometry for comparison by means of the curve of growth.

The resulting length of the radiating volume is between 10 and $70 \mathrm{~m}$, and it clearly depends on the actual brightness of the fireball. Extrapolating, a 10-m long volume of radiating gas is typical for about -1 magnitude and $100 \mathrm{~m}$ is typical for about -12 magnitude. It should be mentioned that the exceptional spectrum, S 1132, has values of $t_{R}$ and $\ell$ smaller than for the other meteors.

The diameter $2 R$ of the radiating volume (for $E_{1}=0$ ) is between $0.4 \mathrm{~m}$ and $2.6 \mathrm{~m}$ and clearly depends on the brightness of the object at the point examined (see fig. 6). A diameter of $28 \mathrm{~cm}$ seems to be typical for 0 magnitude and a diameter of $2 \mathrm{~m}$ typical for -10 absolute magnitude. All these values look reasonable. The values of the characteristic velocity were computed assuming 


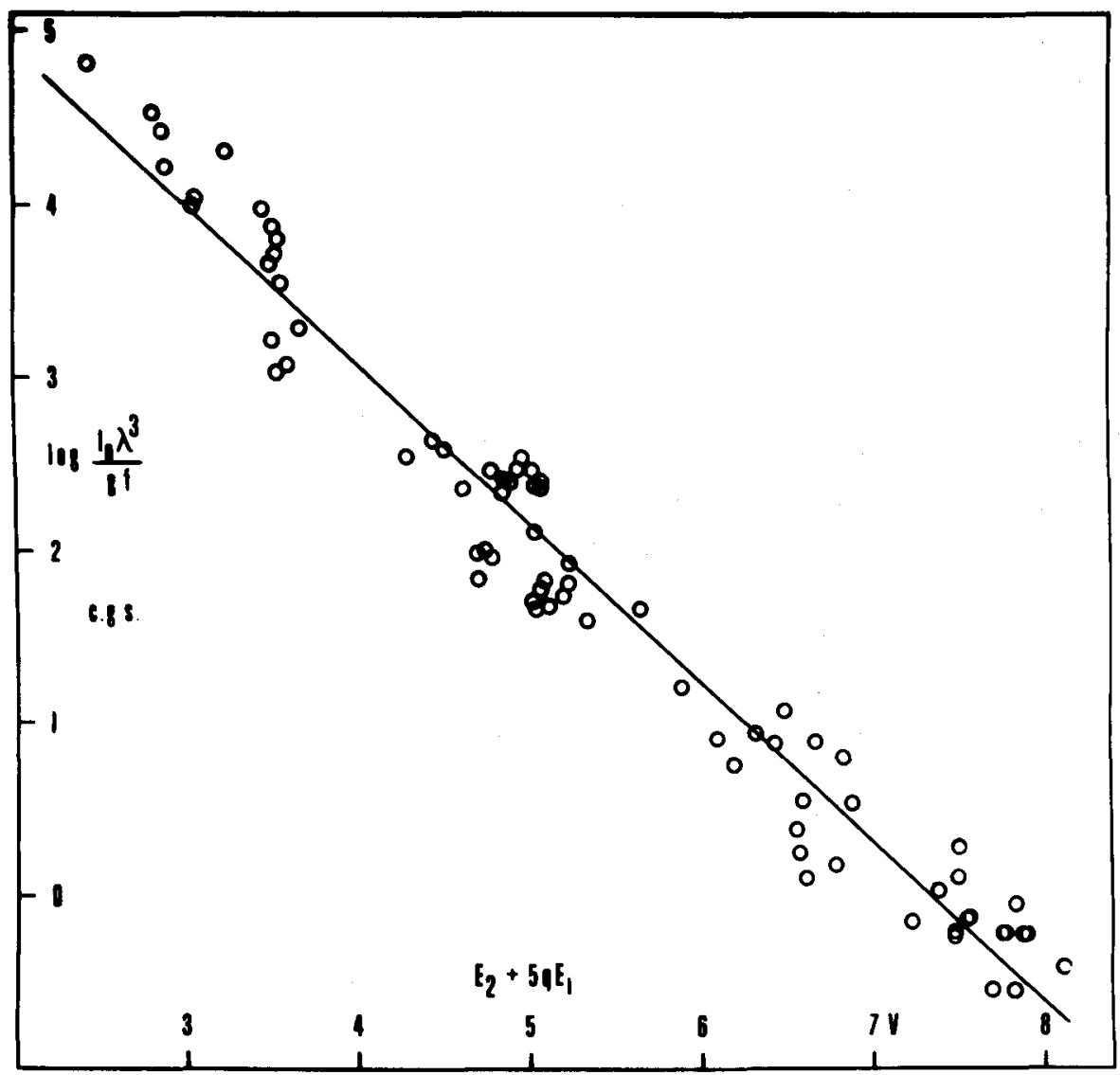

Figure 5.-Spectrum S 1132: The Boltzmann distribution with respect to the decrease in volume of radiating gas with the potential $E_{1}$. The slope of the resulting average straight line defines the temperature, which is used for computation of the curve of growth in figure 4. (All numbers in the abscissae (V) scale should be increased by one.)

that the atoms moved only along the direction of the meteor motion. The resulting characteristic velocities, $v_{m}$, of several $\mathrm{km} / \mathrm{s}$ in most cases, are also what one would expect as the zigzag path of the atom means a greater actual velocity than $v_{m}$. The resulting densities of the iron atoms, if compared with the standard air densities (1962) at corresponding heights, are also quite reasonable.

I tried also to use an exponential change of $\ell$ with the upper potential $E_{2}$. Expressions analogous to equations (16), (17) and (18) were derived, but in this case the numerical solutions resulted in excitation temperatures too high to be admissible for Fe I atoms. Very high densities of the radiating gas relative to the surrounding air point to a great discrepancy in this case. It is clear that the as- sumption of a dependence of the radiating volume on $E_{2}$ potentials is not able to explain the discrepancy between the total light and the spectral data.

Values in table 2 should be considered as one set of possible values only. We chose the ratio $p$ as a constant value. If $p$ is taken as a function of $E_{1}$ and of time, one can obtain solutions which fit the observed points even better, but the number of free parameters is then too high. Also other models than that published in this paper can be used, and thus slightly different expressions for the $x$ - and $y$-axes of the curve of growth and for the $x$-axis of the Boltzmann distribution, can be obtained. Perhaps some solutions may be found better fitting the observational points with such models. 
TABLE 2.-Results for Fe I from Emission Curve-of-Growth With Respect to Changes of the Volume of Radiating Gas with the $\mathrm{E}_{1}$ potential

\begin{tabular}{|c|c|c|c|c|c|c|c|c|c|c|c|}
\hline \multirow[b]{2}{*}{ Spectrum } & \multirow[b]{2}{*}{ Point ${ }^{\mathrm{a}}$} & \multirow{2}{*}{$\begin{array}{c}h \\
(\mathrm{~km})\end{array}$} & \multirow{2}{*}{$\begin{array}{l}{ }^{\mathrm{b}} \log \rho \\
\left(\mathrm{g} / \mathrm{cm}^{3}\right)\end{array}$} & \multirow{2}{*}{$\begin{array}{c}v \\
(\mathrm{~km} / \\
\mathrm{s})\end{array}$} & \multicolumn{3}{|c|}{$\begin{array}{l}\text { Fe I total light } \\
\text { curve data }\end{array}$} & \multicolumn{4}{|c|}{$\begin{array}{l}\text { Fe I emission-curve-of-growth data } \\
\text { for } E_{1}=0\end{array}$} \\
\hline & & & & & ${ }^{c} M_{\mathrm{pan}}$ & $\begin{array}{c}\text { Part } \\
\text { from all } \\
\text { radiation } \\
(\%)\end{array}$ & $\begin{array}{c}\mathrm{d} d m / \\
d t \\
(\mathrm{~g} / \mathrm{s})\end{array}$ & $\begin{array}{c}\text { No. } \\
\text { of } \\
\text { obs. }\end{array}$ & $\begin{array}{c}T \\
\left({ }^{\circ} \mathrm{K}\right)\end{array}$ & $q$ & $\begin{array}{l}\log N \\
\left(E_{1}=0\right)\end{array}$ \\
\hline $\mathrm{S} 6$ & & 76 & -7.4 & 29 & -9.98 & 64 & 3.31 & 88 & $5540 \pm 160$ & 0.041 & $23.70 \pm 0.13$ \\
\hline \multirow[t]{9}{*}{ S 526} & D) & 73.1 & -7.24 & 28.3 & -6.41 & 53 & 1.90 & 37 & $5500 \pm 270$ & 0.045 & $22.78 \pm 0.19$ \\
\hline & $\mathrm{E}$ & 72.7 & -7.22 & 28.1 & -7.42 & 37 & 2.31 & 57 & $5510 \pm 240$ & 0.051 & $22.90 \pm 0.17$ \\
\hline & $\mathrm{F}$ & 72.1 & -7.18 & 28.0 & -7.71 & 49 & 2.43 & 60 & $5510 \pm 230$ & 0.064 & $22.84 \pm 0.17$ \\
\hline & G & 71.7 & -7.16 & 27.9 & -8.49 & 35 & 2.74 & 79 & $5510 \pm 200$ & 0.068 & $23.10 \pm 0.16$ \\
\hline & $\mathrm{H}$ & 71.2 & -7.13 & 27.8 & -7.24 & 51 & 2.25 & 56 & $5500 \pm 220$ & 0.056 & $22.66 \pm 0.16$ \\
\hline & I & 70.6 & -7.09 & 27.6 & -4.70 & 43 & 1.24 & 16 & $5460 \pm 190$ & 0.031 & $21.56 \pm 0.13$ \\
\hline & $J$ & 70.1 & -7.06 & 27.5 & -8.55 & 37 & 2.78 & 76 & $5520 \pm 230$ & 0.060 & $23.10 \pm 0.17$ \\
\hline & $\mathrm{K}$ & 69.2 & -7.01 & 27.2 & -6.15 & 46 & 1.83 & 38 & $5470 \pm 220$ & 0.031 & $22.49 \pm 0.15$ \\
\hline & $(\mathbf{M}$ & 68.6 & -6.98 & 26.9 & -3.75 & 36 & 0.88 & 10 & $5500 \pm 240$ & 0.010 & $20.33 \pm 0.16)$ \\
\hline \multirow[t]{7}{*}{ S 912} & $\mathrm{D}$ & 77.2 & -7.51 & 31.7 & -7.32 & 100 & 2.17 & 22 & $5500 \pm 450$ & 0.012 & $22.42 \pm 0.29$ \\
\hline & $\mathrm{E}$ & 76.3 & -7.45 & 31.6 & -7.76 & 100 & 2.34 & 30 & $5520 \pm 410$ & 0.024 & $22.70 \pm 0.27$ \\
\hline & $\mathrm{F}$ & 75.7 & -7.41 & 31.5 & -8.32 & 100 & 2.57 & 38 & $5470 \pm 370$ & 0.024 & $22.81 \pm 0.25$ \\
\hline & G & 74.8 & -7.35 & 31.3 & -8.68 & 100 & 2.72 & 50 & $5470 \pm 350$ & 0.034 & $22.90 \pm 0.24$ \\
\hline & $\mathrm{H}$ & 74.0 & -7.30 & 30.8 & -10.24 & 95 & 3.36 & 115 & $5520 \pm 190$ & 0.046 & $23.67 \pm 0.15$ \\
\hline & I & 73.9 & -7.29 & 30.6 & -10.10 & 100 & 3.31 & 108 & $5520 \pm 180$ & 0.044 & $23.57 \pm 0.14$ \\
\hline & $\mathbf{J}$ & 73.5 & -7.27 & 30.0 & -10.22 & 100 & 3.37 & 115 & $5500 \pm 190$ & 0.046 & $23.77 \pm 0.14$ \\
\hline S 1132 & & 77.0 & -7.49 & 32.1 & -12.00 & 67 & 4.03 & 75 & $5490 \pm 130$ & 0.055 & $23.65 \pm 0.12$ \\
\hline
\end{tabular}

But the main purpose of this paper is to demonstrate that it is possible to use a simple model of fireball radiation which agrees well with both the total light efficiency and the spectral distribution of the light in absolute values. And this is adequately verified by the values of table 2 .

\section{RESULTS FOR OTHER ELEMENTS THAN FE I}

With a fow exceptions, the whole procedure used for Fe I lines could not be repeated for lines of other elements. The main reason is the low number of lines available for an independent solution.

A solution for $7 \mathrm{Na}$ I lines of spectrum S 1132 was possible with the result $T=5670^{\circ} \pm 170^{\circ} \mathrm{K}$ and $Y_{\text {lim }}=1.4$ for $q=0.05$. The curve of growth starts from the region of strong self-absorption and continues to the damping region. Thus no reliable value of total number of $\mathrm{Na} I$ atoms inside the corresponding radiation volume could be determined. But even if this is not the case, the unknown value of the luminous efficiency factor of sodium prevents one from estimating the relaxation time of $\mathrm{Na}$ I. The dimensions of the radiating volume of $\mathrm{Na} I$ were certainly less than that of Fe $\mathrm{I}$ as is clear from $Y_{1 \mathrm{im}}$ being 1.2 less in log for $\mathrm{Na} I$ than for Fe I. Thus about 4 times less linear dimensions of the radiation volume are found for $\mathrm{Na} I$ atoms than for $\mathrm{Fe}$ I.

A solution for $7 \mathrm{Ca}$ I lines of spectrum S 1132 was also possible with the result $T=4750^{\circ} \mathrm{K} \pm 300^{\circ}$ and $Y_{\text {lim }}=2.6$ for $q=0.05$. The dimensions of the radiating volume are the same as for iron in this case. A slightly better position of the emission curve of growth than for $\mathrm{Na} I$ makes possible an estimation of the number of $\mathrm{Ca} I$ atoms in the whole radiating volume: $\log N=20.8$ and $\log m_{G}=$ -1.4 , which is about 0.1 percent of the $\mathrm{Fe} I$ gas mass.

A solution for 10 multiplets of O I of spectrum S 1132 was possible with a rough result of temperature $19000^{\circ} \pm 4000^{\circ} \mathrm{K}$ and $Y_{\mathrm{lim}}=0.6$ for 
TABLE 2.-Results for F'e I from Emission Curve-of-Growth with Respect to Changes of the Volume of Radiating Gas with the $\mathrm{E}_{1}$ Potential-Continued

\begin{tabular}{|c|c|c|c|c|c|c|c|c|c|c|c|c|}
\hline \multirow{2}{*}{ Spectrum } & \multirow{2}{*}{ Point ${ }^{\mathrm{a}}$} & \multicolumn{11}{|c|}{ Fe I emission-curve-of-growth data for $E_{1}=0$} \\
\hline & & $\begin{array}{l}\log m_{G} \\
(\mathrm{~g})\end{array}$ & $\begin{array}{l}{ }^{\mathrm{f}} Y_{1 \mathrm{im}} \\
\left(\mathrm{cm}^{2}\right)\end{array}$ & $\begin{array}{c}\log t_{R} \\
\text { (s) }\end{array}$ & $\log p$ & $\begin{array}{r}\log R \\
(\mathrm{~cm})\end{array}$ & $\begin{array}{l}\log \ell \\
(\mathrm{cm})\end{array}$ & $\begin{array}{l}\log v_{m} \\
(\mathrm{~cm} / \mathrm{s})\end{array}$ & $\begin{array}{l}\log n_{0} \\
\left(\mathrm{~cm}^{-3}\right)\end{array}$ & $\begin{array}{c}\log \delta_{0} \\
\left(\mathrm{~g} / \mathrm{cm}^{3}\right)\end{array}$ & $\begin{array}{c}\log \bar{n} \\
\left(\mathrm{~cm}^{-3}\right)\end{array}$ & $\begin{array}{c}\log \bar{\delta} \\
\left(\mathrm{g} / \mathrm{cm}^{3}\right)\end{array}$ \\
\hline S 6 & & 1.67 & 2.4 & $-1.64 \mid$ & 1.81 & 1.95 & 3.75 & 5.39 & 14.82 & -7.21 & 15.55 & -6.48 \\
\hline \multirow[t]{9}{*}{ S 526} & $\mathrm{D}$ & 0.75 & 1.2 & -1.15 & 1.81 & 1.73 & 3.54 & 4.69 & 14.56 & -7.47 & 15.28 & -6.75 \\
\hline & $\mathrm{E}$ & 0.87 & 1.5 & -1.44 & 1.81 & 1.73 & 3.54 & 4.98 & 14.67 & -7.36 & 15.39 & -6.64 \\
\hline & F & 0.81 & 1.7 & -1.62 & 1.81 & 1.74 & 3.55 & 5.17 & 14.59 & -7.44 & 15.31 & -6.72 \\
\hline & $\mathrm{G}$ & 1.07 & 1.9 & -1.67 & 1.81 & 1.79 & 3.60 & 5.27 & 14.70 & -7.33 & 15.43 & -6.60 \\
\hline & $\mathrm{H}$ & 0.63 & 1.5 & -1.62 & 1.81 & 1.68 & 3.48 & 5.10 & 14.60 & -7.43 & 15.33 & -6.70 \\
\hline & I & 0.47 & 1.15 & -1.71 & 1.81 & 1.53 & 3.33 & 5.04 & 13.94 & -8.09 & 14.67 & -7.36 \\
\hline & $\mathbf{J}$ & 1.07 & 1.8 & -1.71 & 1.81 & 1.74 & 3.55 & 5.26 & 14.84 & -7.19 & 15.56 & -6.47 \\
\hline & $\mathrm{K}$ & 0.46 & 1.1 & -1.37 & 1.81 & 1.62 & 3.43 & 4.80 & 14.59 & -7.44 & 15.31 & -6.72 \\
\hline & $(\mathbf{M}$ & -1.70 & 1.1 & -2.58 & 1.81 & 1.22 & 3.03 & 5.61 & 13.64 & -8.39 & 14.36 & $-7.67)$ \\
\hline \multirow{7}{*}{ S 912} & 1) & 0.39 & 2.0 & -1.78 & 1.81 & 1.83 & 3.63 & 5.41 & 13.91 & -8.12 & 14.64 & -7.39 \\
\hline & $\mathrm{E}$ & 0.67 & 2.1 & -1.67 & 1.81 & 1.89 & 3.70 & 5.37 & 13.99 & -8.04 & 14.72 & -7.31 \\
\hline & $\mathrm{F}$ & 0.78 & 2.2 & -1.79 & 1.81 & 1.89 & 3.69 & 5.49 & 14.12 & -7.91 & 14.84 & -7.19 \\
\hline & G & 0.87 & 2.3 & -1.85 & 1.81 & 1.90 & 3.71 & 5.56 & 14.17 & -7.86 & 14.89 & -7.14 \\
\hline & $\mathrm{H}$ & 1.64 & 2.5 & -1.72 & 1.81 & 2.01 & 3.82 & 5.54 & 14.60 & -7.43 & 15.33 & -6.70 \\
\hline & I & 1.54 & 2.4 & -1.77 & 1.81 & 1.96 & 3.77 & 5.54 & 14.65 & -7.38 & 15.38 & -6.65 \\
\hline & J & 1.74 & 2.4 & -1.63 & 1.81 & 2.01 & 3.81 & 5.45 & 14.72 & -7.31 & 15.44 & -6.59 \\
\hline S 1132 & & 1.62 & 2.6 & -2.41 & 1.20 & 2.12 & 3.32 & 5.73 & 14.86 & -7.17 & 15.59 & -6.44 \\
\hline
\end{tabular}

Notation of points: see the corresponding paper.

b $\log \rho$ air densities from the U.S. Standard Atmosphere, 1962.

c $M_{\text {pan }}$ is close to absolute panchromatic magnitude, found by comparing stars with meteor spectrum by means of panchromatic emulsion.

$\mathrm{d} \log \tau_{\mathrm{pan}}=-11.94$.

e No. of obs. is the number of Fe I spectral lines used for the solution.

i $Y_{\mathrm{lim}}=\left(\log \left(I /\left(\lambda B_{\lambda}\right)+4 q \theta E_{1}\right)\right.$ limit for optically thick radiation.

$q=0.05$. The linear dimensions of the radiating volume of $\mathrm{O} I$ atoms are 10 times less than that of Fe I. The number of $\mathrm{O} I$ atoms inside this volume is $\log N \approx 20.4$, giving $\log m_{G} \approx-2.2$ (g). A rough estimate of the corresponding atoms of $\mathrm{Fe} \mathrm{I}$ inside the same small volume gives about 1 to 10 percent of $\mathrm{O}$ I mass relative to iron mass.

No other solutions or even rough estimates for all the detailed meteor spectra of table 1 were found. In my previous papers, I used a method of estimating the abundances of other elements than iron from the curve of growth constructed for Fe I and applied to a small number of lines, the only ones available for the corresponding element. But this is possible only on the assumption that (a) the excitation temperature $T$ is the same as that for $\mathrm{Fe} \mathrm{I}$; (b) the dimensions of the radiating volume and the characteristic velocity are the

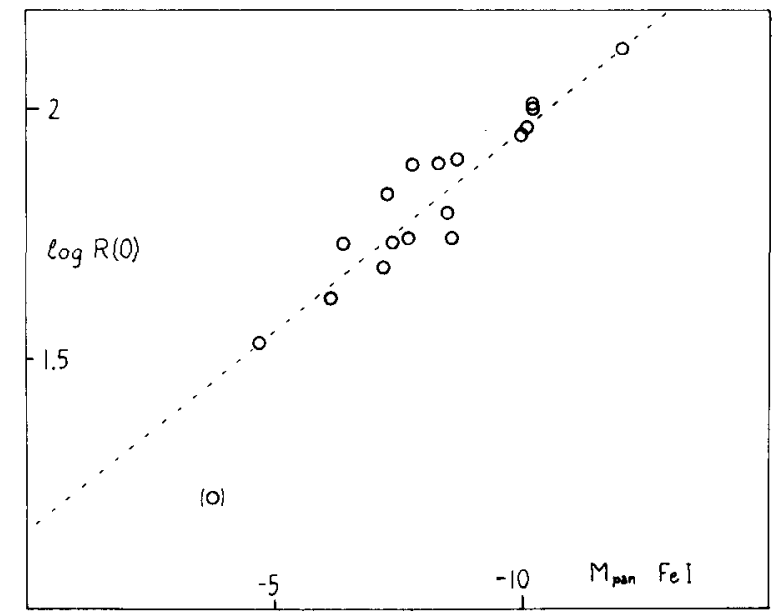

Figure 6.-The dependence of the radiating-volume radius $(\log R)$ of $\mathrm{Fe} I$ on the total actual brightness of Fe $I$ as derived from the spectral analysis of fireballs with $30 \mathrm{~km} / \mathrm{s}$ velocity. 
same as that for $\mathrm{Fe} \mathrm{I}$; (c) the damping constant is the same as that for iron. The independent solutions given above even if only rough guesses, point clearly to the non-validity of all three assumptions. Nevertheless, I tried to find some abundances of other elements than iron by assuming that the prepositions (a), (b) and (c) are valid. The results were very frustrating.

In summary, it does not seem possible to determine any realistic abundances of other elements than Fe I for fireballs from -4 to -12 magnitude. The radiation we receive from these fireballs originates mainly from the effective surface of the radiating volume, and we do not see most of the light originating inside the volume. Thus we are able only to compare some total numbers of atoms radiating from a completely unknown surface of the radiating volume. This also strongly depends on what line of what element we observe. The situation should be somewhat better for meteors as faint as $\mathbf{0}$ magnitude, but this is necessary to check by construction of the emission curve-of-growth for each particular case. As mentioned before, the optical thickness still seems to be an important factor for most spectral lines of 0 magnitude meteors.

\section{CONCLUSIONS}

(a) The radiation of fireballs $(v \approx 30 \mathrm{~km} / \mathrm{s}$, $h \approx 70$ to $80 \mathrm{~km}, M_{\mathrm{pan}} \approx-4$ to $-12 \mathrm{mag}$ ) is strongly affected by self-absorption and to some extent by damping.

(b) Self-absorption should still be quite significant at 0 absolute magnitude. Optically thin radiation may be expected for meteors fainter than +5 absolute magnitude.

(c) If iron lines of short-duration flares are studied by the emission curve of growth and the resulting absolute abundances compared with the total luminosity by means of the luminosity equation factor, the resulting relaxation time is longer than the duration of the flare.

(d) If we assume that the dimensions of the volume of radiating gas decrease with increasing lower potential $E_{1}$, a relaxation time shorter than the duration of the flare can be found. In this way, the spectral data on Fe I lines can be made to agree with the luminous efficiency of the total Fe I meteor radiation. (e) Parameter $q$, relating the dimensions of the radiating volume to the lower potential $E_{1}$, was found partly dependent on meteor brightness. The effect of change of the radiating volume with potential $E_{1}$ is negligible for meteors fainter than about -2 to -1 absolute magnitude.

(f) Excitation temperatures $T \approx 5500^{\circ} \mathrm{K}$ were found to be an optimum solution for $\mathrm{Fe} I$ in all the fireball spectra studied. This is effectively a lower limit, since somewhat higher values are also possible.

(g) A relaxation time of $0.02 \mathrm{~s}$ was found as a typical value, independent of the actual brightness of the fireball. The light of fireballs is emitted during a relatively long relaxation time, which is many orders of magnitude longer than the time necessary for spontaneous radiation of excited Fe I atoms.

(h) The dimensions of the radiating gas volume depend on the fireball brightness. Fe I gas: $0.3 \times 9 \mathrm{~m}$ at 0 absolute magnitude, $2 \times 60 \mathrm{~m}$ at -10 absolute magnitude.

(i) The terminal densities at the end of the radiating volume of iron vapor roughly correspond to the standard air densities at the corresponding heights.

(j) Practically, it was not possible to determine any realistic abundances of other elements than iron for the fireballs studied. A few lines are available for the same independent procedure as used for FeI. The curve of growth derived for Fe I cannot be used for other elements. The radiation we receive from fireballs originates mainly from the effective surface of the radiating volume, which prevents a sufficiently accurate comparison for various elements of the total numbers of atoms inside the whole volume.

(k) The relaxation time and other problems contained in this paper could be studied much easier, if meteor spectra with a very short exposure time (instantaneous exposure) were available.

\section{ACKNOWLEDGMENT AND ERRATUM}

My thanks are due to R. E. McCrosky, who pointed out a discrepancy in the photometric data published in my paper (1966) on an iron meteoroid. (Private communication and McCrosky, 1968.) In preparing this paper I checked all the numbers in the $\mathrm{S} 912$ photometry and found a mistake of exactly 1 in the logarithm of the factor converting from magnitudes to the absolute scale of $I$ in 
$\mathrm{erg} / \mathrm{s}$. In the phrase " 3.59 magnitude $\equiv 2.73$ times less intensity than 0 magnitude" the value 2.73 should be 27.3 .

All data in my 1966 paper given in magnitudes or "bolometric" magnitudes are $\mathrm{OK}$, all data given in $\log I$ $(\mathrm{erg} / \mathrm{s})$ must be corrected by subtraction of 1.00 . Table 6 starts correctly with 11.91 for $\log I$ of point $H$ of line No. 1 and so on till 12.85 for $\log I$ of point $J$ of line No. 189. In figure 2: the $y$-axis must be changed from 12.5 to 11.5 and from 13.5 to 12.5 .

The results in my 1967 paper were derived using the wrong numbers mentioned above, but the systematic error in the absolute values of the oscillator strengths of Fe I acted in the opposite direction. In any case, the problem of the analysis of meteor spectra has been studied from new and more general aspects in this paper, using the correct $\log I$ for S 912 .

In my previous papers, I used the notation "bolometric" magnitudes, to indicate that bolometric magnitudes of stars were used for computation of the absolute c.g.s. scale of intensities. However, the meteor spectral lines were compared with stars using a panchromatic emulsion, and it is usual to speak about "panchromatic" magnitudes in such a case, particularly, if A O comparison stars are used.

\section{LIST OF MATHEMATICAL SYMBOLS}

$\alpha$

$B_{\lambda}$

c

$\delta_{0}$

$\bar{\delta}$

e

$E_{1}$

$E_{2}$

$F(X)$

$f$

$g \equiv g_{1}$

$G_{2}$

$h$

$\chi_{1}$

I

$I_{0}$

$k$

$k_{B}$

$\ell$

$\lambda$
Angle between the line of vision and the meteor motion

Planck's function related to wavelength units

Velocity of light

Terminal density of the radiating volume $\left(\mathrm{g} / \mathrm{cm}^{3}\right)$ radiating volume $\left(\mathrm{g} / \mathrm{cm}^{3}\right)$

Charge of electron

The lower level potential $(\mathrm{V})$

The upper level potential $(\mathrm{V})$

Function giving the curve of growth

Absorption oscillator strength

Statistical weight of the lower level

Total statistical weight of the upper term

Planck's constant

Energy of the lower level

Intensity of optical meteor radiation

Optically thin intensity

Luminous efficiency of the radiating gas per unit gas mass

Boltzmann's constant

Length of the radiating volume

Wavelength

$q$

$t$

$\tau$

$v$
Average density at the end of the $R$

$\begin{array}{ll}m_{\infty} & \text { Meteor mass } \\ m_{\infty} & \text { Initial meteor mass } \\ m_{A} & \text { Total gas mass, which entered into the } \\ & \text { radiating volume from the be- } \\ & \text { ginning of the meteor luminous } \\ & \text { trajectory till time } t \\ & \text { Mass of electron } \\ m_{e} & \text { Total gas mass inside the radiating } \\ m_{G} & \text { volume } \\ & \text { Total gas mass which left the radiating } \\ m_{Z} & \text { volume from the beginning of the } \\ & \text { meteor's luminous trajectory till } \\ & \text { time } t \\ \mu & \text { Mass of one atom } \\ n_{0} & \text { Terminal number of atoms per } \mathrm{cm}^{3} \text { at } \\ & \text { the end of the radiating volume } \\ \bar{n} & \text { Average number of atoms per } \mathrm{cm}^{3} \text { of } \\ & \text { the radiating volume } \\ N & \text { Total number of atoms of one element } \\ & \text { in one state inside the radiating } \\ N \text { (term) } & \text { Tolume } \\ & \text { Total number of atoms being in all } \\ & \text { upper levels of one term inside the } \\ p & \text { radiating volume }\end{array}$

$t_{f}$

$t_{R}$

$T$

$\Theta=\frac{5040}{T}$

$U$

$v_{m}$

$X$

$Y_{\lim }$

Exponential factor giving the decrease of the length of the radiating volume with increasing potential $E_{1}$, definition (14).

Radius of the radiating cylindrical volume

Time

Duration of a flare

Relaxation time of meteor radiation: time spent by an average atom inside the volume of radiating gas

Excitation temperature

Luminous efficiency factor

Boltzmann temperature

Partition function

Meteor velocity

Characteristic velocity

$x$-axis of the curve of growth

Limit value of $y$-axis of the curve of growth for very strong self-absorption 


\section{REFERENCES}

Allen, C. W., 1963. Astrophysical quantities, The Athlone Press, London.

Anon, 1962. U.S. standard atmosphere, 1962, U.S. Committee on Extension of the Standard Atmosphere Supt. of Documents, Washington.

Ayers, W. G., McCrosky, R. E., And Shao, C. -Y., 1970. Photographic observations of 10 artificial meteors, Smithson. Astrophys. Obs. Spec. Rept., No. 317.

Ceplecha, Z., 1964. Study of a bright meteor flare by means of emission curve of growth, Bull. Astron. Inst. Czech., 15, 102-112.

—, 1965. Complete data on bright meteor 32281, Bull. Astron. Inst. Czech., 16, 88-101.

, 1966. Complete data on iron meteoroid (meteor 36221), Bull. Astron. Inst. Czech., 17, 195-206

- , 1967. Spectroscopic analysis of iron meteoroid radiation, Bull. Astron. Inst. Czech., 18, 303-310.

—, 1968. Meteor Spectra, in Physics and Dynamics of Meteors, edited by L. Kresák and P. M. Millman, D. Reidel Publ. Co., Dordrech, Holland, 73-83.

- 1971. Spectral data on terminal flare and wake of double-station meteor No. 38421, Bull. Astron. Inst. Czech., 22, 219-304.

Ceplecha, Z. AND RAJChL, J., 1963. The meteor spectrum with dispersion from 11 to $38 \AA / \mathrm{mm}$, Bull. Astron. Inst. Câech., 14, 29-49.

Corliss, C. H. AND Bozman, W. R., 1962. Experimental transition probabilities for spectral lines of seventy elements, NBS Monograph 53, Supt. of Documents, Washington.

Corliss, C. H. AND Warner, B., 1964. Absolute oscillator strengths for Fe I, Astrophys. J. Suppl. Ser., 8, 395.

Fritchtenicht, J. F., Slattery, J. C., and Tagliaferri, E., 1968. A laboratory measurement of meteor luminous efficiency, Astrophys. J., 151, 747-758.

Garz, T. AND Kock, M., 1969. Experimentelle Oszillatorenstärken von Fe I-Linien, Astron. Astrophys., 2, 274-279.

Grasdalen, G. L., Huber, M., and Parkinson, W. H., 1969. Absolute $g f$-values for Fe I and and Fe II lines, Astrophys. $J$., 156, 1153-1173.

Harvex, G. A., 1970. Spectra of faint optical meteors, presented at 13th Plenary Meeting of COSPAR, Leningrad, unpublished.

McCrosky, R. E., 1968. Meteors without sodium, Smithson. Astrophys. Obs. Spec. Rept. No. 270.

Moore, C. E., 1945. A multiplet table of astrophysical interest, Contrib. Princeton Obs. No. 20.

Wiese. W. L., Smith, M. W., and Clennon, B. M., 1966. Atomic transition probabilities, $\mathrm{Na-}$ tional Standard Reference Data Series, NBS 4, Washington.

Wiese, W. L., Smith, M. W., ANd Miles, B. M., 1969. Atomic transition probabilities, National Standard Reference Data Series, NBS 22, Washington. 